\title{
ESTIMATE OF RAIN EVAPORATION RATES FROM DUAL-WAVELENGTH LIDAR MEASUREMENTS: COMPARISON AGAINST A MODEL ANALYTICAL SOLUTION
}

\author{
Simone Lolli ${ }^{1 *}$, Paolo Di Girolamo ${ }^{2}$, Belay Demoz ${ }^{3}$, Xiaowen $\mathrm{Li}^{4}$, Ellsworth J. Welton ${ }^{5}$ \\ ${ }^{1}$ NASA GSFC-JCET, Code 612, Greenbelt, MD, 20771,USA *simone.lolli@nasa.gov \\ ${ }^{2}$ Università della Basilicata, Via Sauro 85, 85100 Potenza, Italy \\ ${ }^{3}$ UMBC-JCET, 1000 Hilltop Circle, 21250 Baltimore, MD, USA \\ ${ }^{4}$ NASA GSFC-GESTAR, Code 612, 20771 Greenbelt, MD, USA \\ ${ }^{5}$ NASA GSFC, Code 612, 20771 Greenbelt, MD, USA
}

\begin{abstract}
Rain evaporation significantly contributes to moisture and heat cloud budgets. In this paper, we illustrate an approach to estimate the median volume raindrop diameter and the rain evaporation rate profiles from dual-wavelength lidar measurements. These observational results are compared with those provided by a model analytical solution. We made use of measurements from the multi-wavelength Raman lidar BASIL.
\end{abstract}

\section{INTRODUCTION}

Rain evaporation, while significantly contributing to moisture and heat cloud budgets, is still a poorly understood process with few measurements presently available. In this paper we describe an application of the color ratio technique $(C R)$ [1] for the estimation of rain evaporation rates based on the use of a dualwavelength lidar, which is an active optical device particularly suited to investigate atmospheric constituents, especially aerosols [2], [3], [4] and clouds [5], [6]. Several authors (among others [8]) reported lidar measurements during light precipitation events, i.e. drizzle and virga. In particular in [1], a technique is developed to retrieve the median volume raindrop diameter profile from the measurements performed by two co-located commercial lidars operating at $355 \mathrm{~nm}$ and $532 \mathrm{~nm}$. This technique allows retrieving the median volume raindrop diameter profile and the evaporation rate, which in turn it is used to validate a model analytical solution [10]

\section{METHODOLOGY}

Several approaches to retrieve the median volume raindrop diameter during light precipitation events using two-wavelength lidar backscatter data have been illustrated by different authors [1], [9]. All these approaches take advantage of the sensitivity of the differential backscattering efficiency at the two wavelengths to the rain Drop Size Diameter, DSD $\left(\mathrm{UV}_{355 \mathrm{~nm}^{-}}\right.$ $\mathrm{VIS}_{532 \mathrm{~nm}}$ in [1], $\mathrm{IR}_{905 \mathrm{~nm}}-\mathrm{IR}_{1500 \mathrm{~nm}}$ in [9]). For example, the approach reported in [1] relies on the consideration that the backscattering efficiency at $532 \mathrm{~nm}$ is much less sensitive to water sphere diameter with respect to the backscattering efficiency at $355 \mathrm{~nm}$ (while the extinction coefficient are wavelength independent). This is depicted in Fig. 1a and 1b, which show how the backscattering efficiencies calculated using an algorithm based on Mie theory for large size parameter values (i.e. for large particles with respect to the sounding wavelength) [1] are dependent on water sphere diameter at $355 \mathrm{~nm}$ (blue dots) and 532nm (green dots). Integrating those values of the backscattering efficiencies (Fig. 1a) over a modified normalized gamma raindrop size distribution (see Eq. 4 in [1]) for $D_{0}=1-1000 \mu \mathrm{m}$, the differences between the backscattering efficiencies in function of the median volume raindrop diameter $D_{0}$ become substantial, allowing to define the color ratio (Fig. 1c) as ten times the logarithm of the ratio of the rain backscattering coefficients at the two wavelengths. From the measured color ratio it is possible retrieving the median volume raindrop diameter at each range bin using the curves in Fig. $1 \mathrm{c}$ as look-up tables. Accurate information on the method is beyond the scope of this paper and can be found in [1]. Subsequently, the evaporation rate can be estimated as the change of the raindrop volume (shrinking/growing) during the 
precipitation along the profile for each two adjacent levels as (Eq. 1):

$$
E(r)=\frac{\left(d_{r+1}\right)^{3}-d_{r}{ }^{3}}{\left(d_{r+1}\right)^{3}}
$$

where the subscripts $r$ and $r+1$ indicate the raindrop diameter $d$ at current range bin $r$ and immediately above $(r+1)$. The evaporation rate is closely related to the humidity content of the surrounding atmosphere and to atmospheric processes as breakdown or coalescence. The raindrop diameter and evaporation rate retrieved by lidar with the method described above can be finally compared with the analytical solution model proposed in [10].

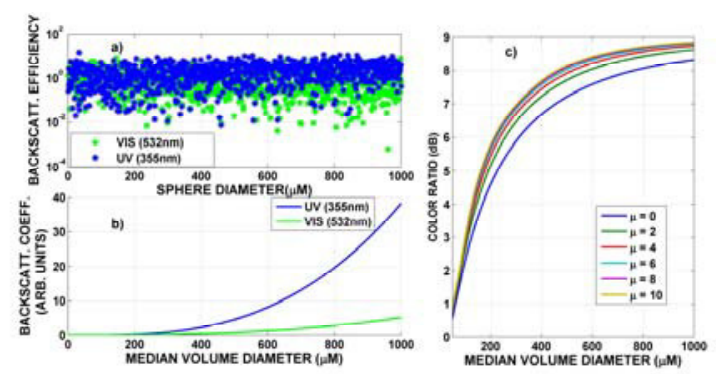

Figurel a): Backscattering efficiencies obtained with Mie code vs. sphere diameter for 532nm (green dots) and $355 \mathrm{~nm}$ (blue dots); b) Backscattering coefficient vs. the median volume at two different wavelengths (355 and 532nm, respectively); c) Color ratio obtained from the ratio of the two rain backscattering coefficients in b) for different values of the shape parameter $\mu$ of the gamma raindrop distribution

\subsection{Analytical Model}

The analytical model determines the "evaporation power" of a stationary atmospheric layer, based on the computation of the single raindrop diameter $\mathrm{D}^{*}$ (dependent only on temperature and water vapor), defined as the diameter of a single raindrop fully evaporating in the selected layer [10]. The model relies on the assumption of a steady environment with negligible vertical air motion. In [10] these conditions were assumed to be valid for stratiform rain, but in the present paper these conditions are extended to selected virga episodes. Instead of calculating $\mathrm{D}^{*}$, in this study we compute the temporal evolution of each single raindrop diameter present in the precipitation at each different atmospheric layer, from cloud base to complete evaporation [10]. The thickness of each layer is defined from the radiosonde spatial resolution. The temporal evolution of the raindrop diameter only depends on the thermodynamic properties of the considered atmospheric layer (measured by the radiosonde). A raindrop diameter $\mathrm{D}_{1}$ at cloud base (at a certain altitude $\mathrm{h}_{1}$ ) evolves into diameter $\mathrm{D}_{2}$, at the bottom of the first considered layer (at a certain altitude $\mathrm{h}_{2}$ ). Then $\mathrm{D}_{2}$ becomes the input diameter of the top of the second considered layer and so on, down to the last available layer before complete evaporation. The thermodynamic properties of the atmospheric layer with a thickness defined by $h_{1}-h_{2}$ are determined from the radiosonde.

\section{RESULTS}

On 23 July 2007 [8], a cyclonic system, developed in the northern Atlantic region, moved toward the COPS measurement site, producing stratiform clouds with consequent stratiform rain occurring in the afternoon. These steady meteorological conditions are optimal to apply the analytical model. The time evolution of the backscattering ratio at $1064 \mathrm{~nm}$ for the time interval 13:00-14:35 UT is shown in Fig. 2. The figure clearly reveals the presence of a lidar dark band [8] around 2.8 $\mathrm{km}$, associated with changes in scattering properties of precipitating hydrometeors taking place in the melting layer during the snowflaketo-raindrop transition. This figure also reveals the presence of stratiform clouds, with cloud base at $3.1-3.3 \mathrm{~km}$ above ground level (a.g.1.). Ancillary information on the thermodynamic state of the atmosphere was provided by ad-hoc radiosondes, launched every three hours during the measurement session [8].

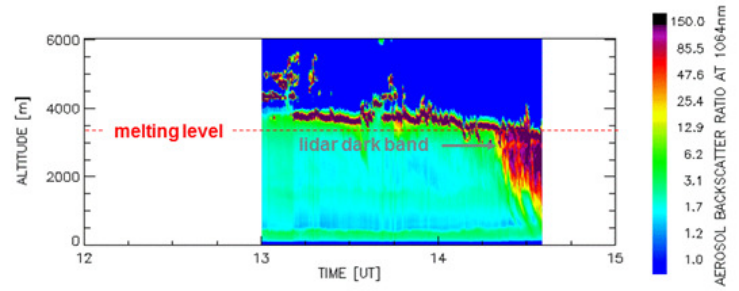

Figure 2 Time evolution of the backscattering ratio at 1064nm for the time interval 13:00-14:35 UT on 23 July 2007 as measured by the lidar system BASIL

The profile of raindrop diameter corresponding to the above estimated $C R$ profile is illustrated in Fig. 3 (blue line), with the relative uncertainty that takes into account also the total error on the measured color ratio. This error includes contributions from the signal measurement 
uncertainty, the two-way transmission error, and the atmospheric density uncertainty. The total $C R$ error is then related to the uncertainty of the measured range-corrected signal that it is, in turn, related to the Poisson photon-counting statistic at each range level; the uncertainty on the molecular backscattering is related to the molecular density profile and for the error in aerosol transmission is assumed to be constant (8\%, [1]) while the error on molecular transmission is related to the again to the molecular density. The median volume raindrop diameter ranges from 100 to $350 \mu \mathrm{m}$.

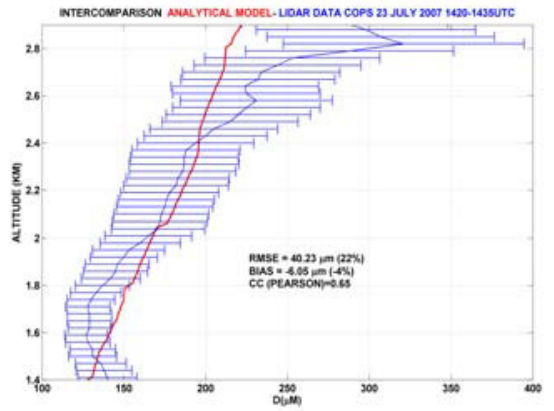

Figure 3: Vertical profile of the median volume raindrop diameter (blue line, with error bar) as determined from the lidar measurements on 23 July 2007 and model analytical solution (red line). The intercomparison shows a 22\% Root Mean Square Error and 17\% Mean Fractional Error with 5\% bias. Regarding the shape, the Pearson Correlation Coefficient shows a positive correlation of $65 \%$.

The model analytical solution for the median volume raindrop diameter $D_{0}$ (also reported in Fig. 3, red line) is calculated assuming constant values for water vapor mixing ratio and temperature within each layer, these values taken from the simultaneous and co-located radiosonde profile (launched from the lidar station at 14:06 UTC on 23 July 2007). In this respect, it is to be noticed that, even when launched from the same location of the lidar, the radiosonde is not perfectly co-located with the vertical column illuminated by the lidar, as in fact the former may undergo horizontal drifts due to the wind during its ascent. However, the radiosonde data considered in this case study indicate an overall horizontal drift not exceeding $1.8 \mathrm{~km}$ at $3 \mathrm{~km}$; this aspect, together with the verification of the very limited variability of the radar reflectivity profiles at 1.29 and $35.5 \mathrm{GHz}$ (not shown here, but illustrated in Fig. 3 and Fig. 4 of [8]) confirms that the air masses sounded by the lidar and the radiosonde are substantially the same. Fig. 4 compares the vertical profile of the evaporation rate obtained from lidar measurements (blue) and from the analytical model solution (red). Lidar data show that evaporation occurs primarily in the first 400-500 m below the cloud base (down to 2.8 $\mathrm{km}$ ), with a median volume raindrop diameter reduction in excess of $20 \%$, possibly associated with mixed phase/melting, while in the remaining portion of precipitation (down to $1.4 \mathrm{~km}$ ) diameter reduction is smaller. It is to be noticed that lidar measurements catch a much larger variability in the evaporation process than the model. Large negative values in the lidar-based estimate of the evaporation rate profile are found between 2.6 and $2.8 \mathrm{~km}$. We can speculate that these large negative values are partially fictitious and attributable to the progression of the melting process, partially masking evaporation. This speculation is confirmed by the aircraft in-situ measurements, which reveal that melting is still progressing in the first 400-500 $\mathrm{m}$ below the cloud base (reported in [8]). We believe that the effect of multiple scattering on these results is negligible. In fact, channels used to measure lidar signals at both 355 and $532 \mathrm{~nm}$ are characterized by a very small field-of-view $(240 \mu \mathrm{rad})$, which minimizes potential signals' contamination by multiple scattering. Additionally, the color ratio is obtained by ratioing these two signals so that, in case of any residual multiple scattering effect on the signals, this cancels out in the color ratio. In order to obtain a proper quantitative assessment of the agreement between the model and the measurements, the root-mean square (RMS) deviation, the bias (BIAS) and the correlation coefficient [3], [11]) of model vs. lidar data have been computed and are found to be $40.23 \mu \mathrm{m}$ $(22 \%),-6.05 \mu \mathrm{m}(-4 \%)$ and 0.65 , respectively. Small values for RMS deviation and BIAS and the large value for the correlation coefficient testify the good agreement between model and lidar results.

\section{CONCLUSIONS}

A comparison between the model analytical solution proposed by [10] and estimates obtained from multi-wavelength lidar data in terms of the median volume raindrop diameter and rain evaporation rate was carried out for two selected 
case studies: one collected on 23 July 2007 by the Raman lidar system BASIL during the COPS experiment. The inter-comparison reveals good agreement between model and observations, with a correlation between the profiles up to $65 \%$, a root mean square error up to $22 \%$ and a bias of $5 \%$. Local higher discrepancies are due to i) the radiosonde soundings different air masses and ii) model assumptions being no longer valid along the profile, as in case of non-steady atmosphere and/or of appearance of collision-coalescence processes.

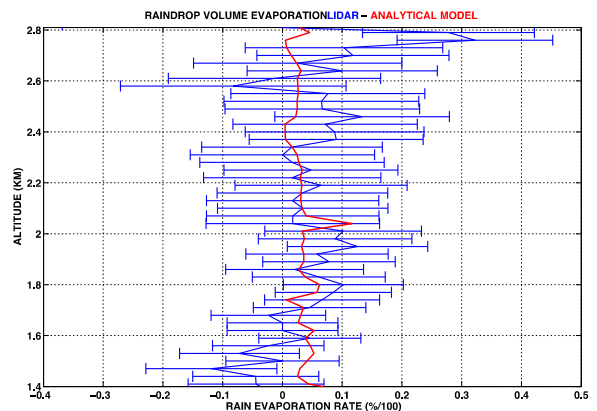

rigure 4: Kalnarop evaporation rate (tn \%/lvu, blue line) and comparison with the model analytical solution (red line). In the first $200 \mathrm{~m}$ below the cloud base the melting process is not completely ended and the rain is mixed with few snowflakes. This determines a strong evaporation (volume shrinkage $>20 \%$ ), which in reality is fictitious. Breakdown is not taken into account by the model. This can explain the difference with the lidar data in the 1.4-1.8 km atmospheric layer. Negative values represent a volume growth of the raindrop

\section{ACKNOWLEDGEMENTS}

This study and the NASA Micro Pulse Lidar Network (MPLNET) are supported by the NASA Radiation Sciences Program (H. Maring).

\section{References}

[1] Lolli S. et al, 2013: Evaluating light rain drop size estimates from multiwavelength micropulse lidar network profiling. J. Atmos. Oceanic Technol., 30, 2798-2807

[2] Di Girolamo, P. et al., 1995: Two wavelength Lidar analysis of stratospheric aerosol size distribution", J. Aeros. Sci, 26, 989-1001, doi: 10.1016/0021-8502(95)00025-8.

[3] Lolli S., et al.,. 2013: 0.355-micrometer direct detection wind lidar under testing during a field campaign in consideration of ESA's ADM-
Aeolus Mission, Atmospheric Measurement Techniques, 6, 3349-3358, doi:10.5194/amt-63349-2013

[4] Tan, F. et al., 2014: Variation In Daytime Troposphereic Aerosol Via LIDAR And Sunphotometer Measurements In Penang, Malaysia AIP Proceedings 1588, 286; doi: 10.1063/1.4866962.

[5] J. Campbell, S. Lolli J. Lewis, Y. Gu, E. Welton, 2016: Daytime Cirrus Cloud Top-ofAtmosphere Radiative Forcing Properties at a Midlatitude Site and their Global Consequence. Journal of Applied Meteorology and Climatology, http://dx.doi.org/10.1175/JAMCD-15-0217.1

[6] S. Lolli, J. Lewis, J. R. Campbell, Y. Gu, E. Welton, 2016, "Cirrus Cloud Radiative Characteristics from Continuous MPLNET Profiling at GSFC in 2012. Óptica pura y aplicada, 49 (1), 1-6.

[7] Demoz, B. et al., 2000: Raman LIDAR Detection of Cloud Base. Geophys. Res. Lett., 27, 1899-1902.

[8] Di Girolamo, et al., 2012: Lidar and radar measurements of the melting layer: observations of dark and bright band phenomena, Atmos. Chem. Phys., 12, 41434157.

[9] Westbrook, C. D et al., 2010: Estimating drizzle drop size and precipitation rate using two-colour lidar measurements. Atmos. Meas. Tech., 3, 671-681, doi:10.5194/amt-3-6712010

[10] Li, X. and Srivastava, R., 2001: An Analytical Solution for Raindrop Evaporation and Its Application to Radar Rainfall Measurements.", J. of Applied Met., 40 (9), 1607-1616

[11] S. Lolli; P. Di Girolamo. 2015: Principal component analysis approach to evaluate instrument performances in developing a costeffective reliable instrument network for atmospheric measurements." Journal of Atmospheric and Oceanic Technology, Vol. 32 (9), 1642-1649. 